\title{
Reduced cognitive dysfunctions in rats when the temporal-entorhinal cortices and hippocampal region are denervated simultaneously
}

\author{
TROND MYHRER and KATRINE WANGEN \\ Norwegian Defence Research Establishment, Kjeller, Nomway
}

\begin{abstract}
Transections of the fiber connections between the temporal cortex (TC) and the lateral entorhinal cortex (LEC) result in decreased ability to retain visual information, whereas transections of the hippocampal perforant path (PP) fibers cause decreased ability to acquire visual information. It was assumed that a combination of these two lesion types would mimic the pathology of some neurogenerative disorders and produce severe impairment in both acquiring and retaining new information. Surprisingly, TC/LEC + PP lesions did not cause any effects in a visual discrimination task, whereas separate TC/LEC or PP lesions affected behavior as described above (Experiment 1). In Experiment 2, a novelty test was employed to validate further the lack of effects seen to follow TC/LEC + PP lesions. Initially, an effect of TC/LEC + PP lesions appeared, but vanished as testing proceeded. Separate TC/LEC or PP lesions resulted in reduced preference for novelty throughout all test sessions. Experiment 3 was undertaken to highlight potential neurochemical mechanisms involved in the compensatory processes apparently initiated by the combined lesions. Relatively enhanced cholinergic activity in the hippocampus was found in TC/LEC + PP animals, whereas glutamatergic measures seemed to be unresponsive. The results are discussed in terms of possible compensatory mechanisms underlying the recovery of function seen in rats bearing TC/LEC + PP transections.
\end{abstract}

Results from recent animal research show that the temporal-entorhinal complex is critically involved in mnemonic functions. In the rat, damage to the perirhinal cortex, which mediates connections between the temporal and entorhinal cortices, results in memory deficits in various behavioral tasks (Mumby \& Pinel, 1994; Myhrer \& Wangen, 1996; Nagahara, Otto, \& Gallagher, 1995; Otto \& Eichenbaum, 1992; Wiig \& Bilkey, 1994a, 1994b).

Figure 1 shows diagrams of horizontal sections of the rat temporal-hippocampal region. The medial entorhinal cortex (MEC) and the lateral entorhinal cortex (LEC) project to the hippocampus proper and fascia dentata by way of the medial and lateral perforant path, respectively (Hjorth-Simonsen, 1972; Hjorth-Simonsen \& Jeune, 1972). Moreover, the connection between LEC and the temporal cortex (TC; Broadmann's area 20,36,41) is partly mediated by way of perirhinal cortex (PC). In the rat, TC projects predominantly to LEC via PC (Deacon, Eichenbaum, Rosenberg, \& Eckman, 1983; Vayssettes-Courchay \& Sessler, 1983). In turn, LEC projects heavily to TC, whereas the contribution of MEC is very modest in this respect (Kosel, Van Hoesen, \& Rosene, 1982; Swanson \& Köhler, 1986). TC is further connected with both neocortical and subcortical structures (Deacon et al., 1983). The perforant path fibers are routed in the angular bundle (Hjorth-

This research was supported by the Research Council of Norway Grant 326.93/010 (T.M.) and the Ring Foundation, Oslo (K.W.). Correspondence should be addressed to T. Myhrer, Norwegian Defence Research Establishment, Division for Environmental Toxicology, P.O. Box 25, N-2007 Kjeller, Norway.
Simonsen, 1972; Hjorth-Simonsen \& Jeune, 1972), and the fiber connections between TC and LEC are located in the adjacent white matter (Deacon et al., 1983; Myhrer, Iversen, \& Fonnum, 1989; Vogt, 1974). The connections of TC seem to be partly mediated in the white matter in a radial pattern (Deacon et al., 1983).

Transections of the connections between TC and LEC (TC/LEC) result in impairment of both proactive memory (anterograde amnesia) and retroactive memory (retrograde amnesia) in rats. A slight deficit in postoperative acquisition of the visual discrimination task used is also seen in the proactive paradigm, but this deficit is probably of mnemonic nature, because the initial phase of the training is unaffected by the lesion. Following TC/LEC lesions degeneration is clearly seen in layer II of LEC (Myhrer, 1989b; Myhrer \& Nævdal, 1989). Entorhinal degeneration is also seen after transections of the perforant path (PP). Total disruptions of PP cause degeneration among the stellate cells in layer II in both MEC and LEC. At the behavioral level, PP transections produce reduced preference for novelty, probably by hampering sensory information of neocortical origin from reaching the hippocampus (Myhrer, 1988b). PP lesions are also seen to impair the acquisition of visual discrimination, but not the retention of this task (Myhrer, 1992).

The entorhinal cortices are among the earliest structures to show neuropathology in Alzheimer's disease, Down's syndrome, and aging (Braak \& Braak, 1991; Levisohn \& Isacson, 1991). Thus, animals with partial neuronal loss in the entorhinal area would probably resemble rather closely the pathology of some neurogenerative disorders. 

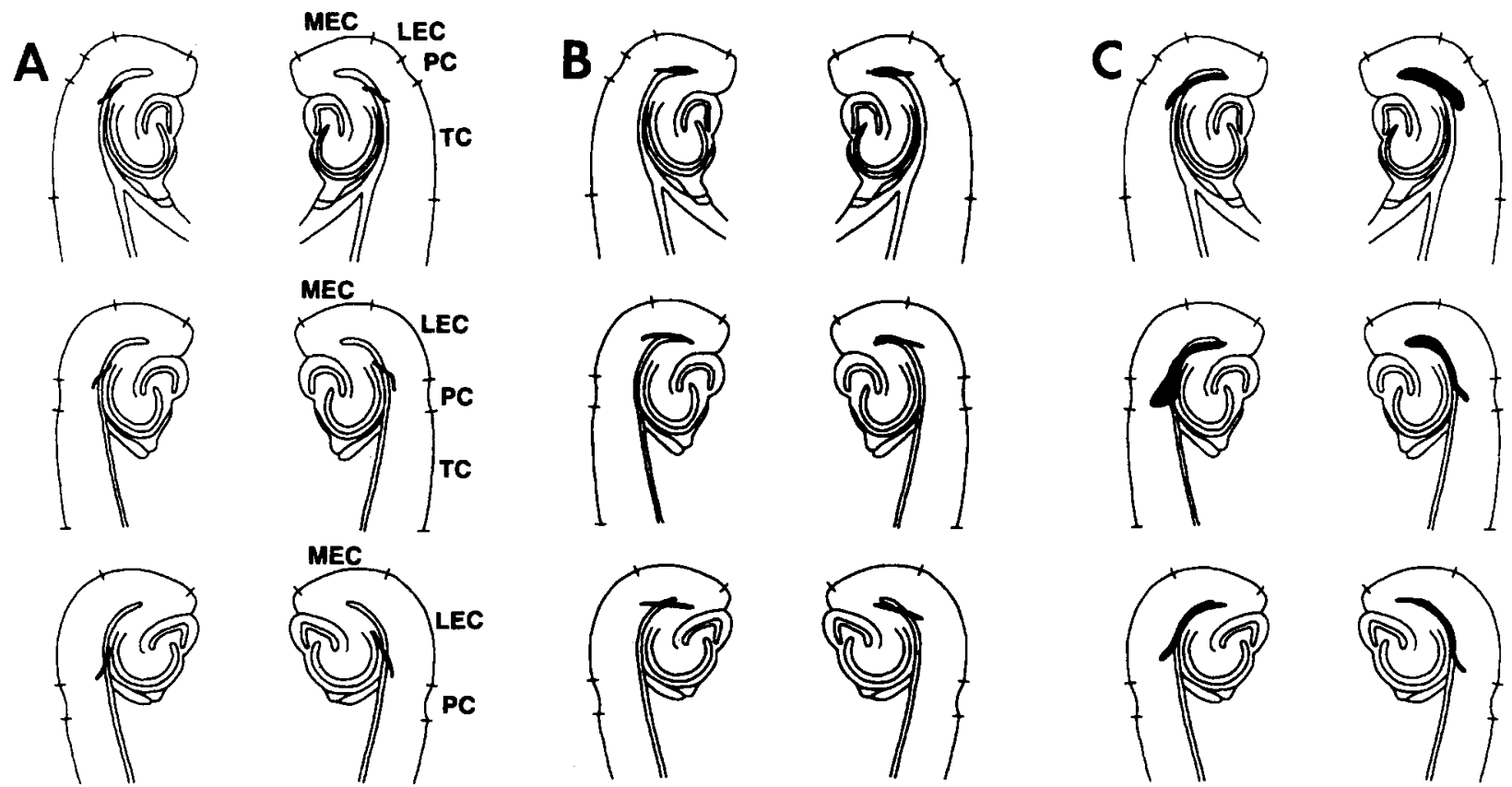

Figure 1. Reconstruction of brain sections indicating locations of the lesions in three levels of horizontal sections. Examples of temporal cortex/lateral entorhinal cortex (TC/LEC) lesion (A), perforant path (PP) lesion (B), and TC/LEC + PP lesion (C). MEC, medial entorhinal cortex; PC, perirhinal cortex.

A combination of TC/LEC and PP lesions would be expected to affect severely the entorhinal cortices and result in impaired abilities to both acquire and retain new information. The purpose of this study was to examine this hypothesis by testing rats with either TC/LEC, PP, or TC/LEC + PP lesions in a three-choice simultaneous brightness discrimination task used in previous studies.

\section{EXPERIMENT 1}

\section{Method}

Subjects. Thirty-six male Wistar rats from a commercial supplier (Møllegaard Breeding Laboratories, Denmark), weighing $290-320 \mathrm{~g}$ at the time of surgery, served as subjects. They were randomly assigned to four groups: 9 rats received bilateral $\mathrm{TC} /$ LEC lesions, 9 received PP lesions, 9 received TC/LEC + PP lesions, and 9 served as controls and had only their scalp reflected. Their group assignment was not known during testing. The rats were housed individually and had free access to commercial rat pellets and water. They were handled individually 3 days preoperatively and 1 day postoperatively, being allowed to explore a table top $(80 \times 60 \mathrm{~cm})$ for $3 \mathrm{~min}$ a day. The climatized $\left(21^{\circ} \mathrm{C}\right) \mathrm{vi}-$ varium was illuminated from 0700 to $1900 \mathrm{~h}$.

Surgery. The rats were anesthetized with diazepam $(10 \mathrm{mg} / \mathrm{kg})$ and fentanyl fluanisone $(2 \mathrm{mg} / \mathrm{kg}$ ) and placed in a stereotaxic head holder with their skulls horizontal. The bilateral TC/LEC lesions were made mechanically by means of the sharp edges of cannulas (diam. $0.5 \mathrm{~mm}$ ) provided with collar to control for insertion depth. The cannula to be used was mounted on a syringe. The point of insertion was $7.8 \mathrm{~mm}$ posterior to bregma and $6.7 \mathrm{~mm}$ lateral to midline. Each cannula was inserted into the brain in a position deviating $20^{\circ}$ from the vertical in the sagittal plane (tip of cannula pointing rostrally). From this position the syringe was moved 10 times back and forth in an axis deviating about $45^{\circ}$ from the frontal plane (opening of angle pointing medially). These maneuvers were carried out in two stages with insertion depths 6 and $8 \mathrm{~mm}$ from top of skull. In this way, the distal part of the angular bundle was transected at a site corresponding approximately to the level of the rhinal fissure.

$\mathrm{PP}$ lesions were made in the same way as described for TC/LEC lesions. The point of cannula insertion was $8.5 \mathrm{~mm}$ posterior to bregma and $5.0 \mathrm{~mm}$ lateral to midline. Each cannula was inserted into the brain in a position deviating $20^{\circ}$ from the vertical in the sagittal plane (tip of cannula pointing rostrally). From this position the syringe was moved 10 times back and forth in the frontal plane, making a cut of $1.0-1.5 \mathrm{~mm}$ through the angular bundle. These maneuvers were carried out in three stages with depth of insertions 4,6 , and $8 \mathrm{~mm}$ from surface of the skull to tip of cannula. The control rats received a corresponding incision in the scalp only.

Histology. Upon termination of testing, the animals were decapitated, and the brains were removed and frozen. The brains were sectioned horizontally on a $\mathrm{CO}_{2}$-freezing microtome at $30 \mu \mathrm{m}$, every 12 th section being preserved. The sections were stained with methylene blue. The extent of fibers transected was estimated from the degree to which the white matter between TC and LEC was damaged at the three dorsoventral levels presented in Figure 1 . The white matter (not the alveus) was divided in four equal columns, each column representing $25 \%$ of the fibers. The occurrence of damage was evaluated under $\times 100$ magnification in a light microscope. The number of columns affected at each dorsoventral level were counted, and the mean percentage of damage was computed for each animal.

The percentage of PP damage was based on the location and extent of the transections in relation to the course of PP fibers. The lesions were traced on diagrams of the PP fibers consisting of 20 lines evenly distributed, each line representing $5 \%$ of the fibers. The number of lines affected in the dorsoventral levels presented in Figure 1 were counted, and the average percentage of damage for each animal was computed. 

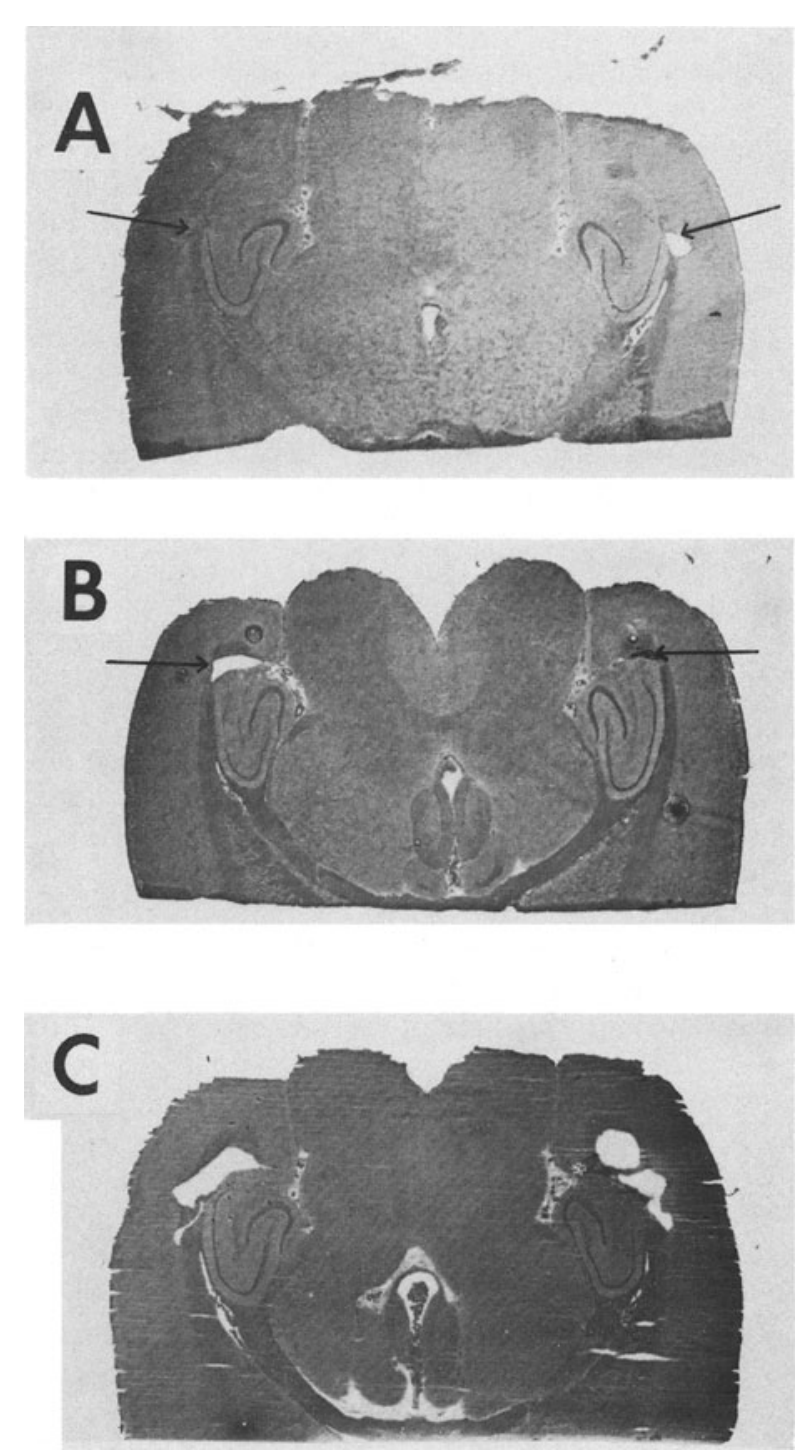

Figure 2. Photographs of horizontal sections showing appearance of temporal cortex/lateral entorhinal cortex (TC/LEC) lesion (A), perforant path (PP) lesion (B), and TC/LEC + PP lesion (C).

Apparatus. Testing of simultaneous brightness discrimination was carried out in a Plexiglas cage $(56 \times 34 \times 20 \mathrm{~cm})$ previously described (Myhrer \& Nævdal, 1989). In brief, a Plexiglas wall with an opening $(10 \times 10 \mathrm{~cm})$ in the middle divided the apparatus into two equal compartments - start compartment and goal compartment. Three interchangeable aluminium cylinders $(3 \times 7 \mathrm{~cm})$ with a round well $(2 \times 2 \mathrm{~cm})$ in the top served as discriminanda. The cylinders were located in fixed positions (equal distance between each) along the wall opposite to the partition wall in the goal compartment. The cylinders were natural gray (aluminium) or painted black (except for the well). The well of the positive cylinder was filled with water. The only light was a $15-\mathrm{W}$ bulb $60 \mathrm{~cm}$ above the apparatus.

Procedure. During acquisition and retention testing, the rats were deprived of water for $231 / 2 \mathrm{~h}$ a day. Prior to aquisition training (Postoperative Day 8), each rat was allowed to explore the empty test apparatus for $15 \mathrm{~min}$. During the first test session, the subjects were trained to run from the start compartment into the goal compartment, in which they were rewarded with some laps of water from the well in the positive cylinder. That is, the rats were permitted to inspect cylinders until they encountered the correct one. The rats were given 10 trials with an intertrial interval of $20 \mathrm{sec}$, during which they stayed in their home cage. During the second test session, the animals were given trials until the occurence of five correct responses in succession. Because the task is rapidly learned, learning criterion was set low to avoid overlearning. Thirteen days after learning criterion had been reached, the animals were tested for retention of the discrimination task. Testing was terminated when the previous criterion was reached. The following behaviors were recorded: number of trials to criterion, and number and type of errors to criterion. In order to drink or investigate whether the well in a cylinder contained water, the rats had to stand on their hind legs with at least one forepaw on top of the cylinder. Error response was scored when a negative cylinder was mounted and found empty of water (e.g., licking the empty well). Approaching or investigating negative cylinders (except the well) was not scored as an error. The positive cylinder was either black or gray, and the two cylinders of opposite color were negative. The position of the positive cylinder (left, middle, right) was changed in a prearranged randomized order. One set of randomized positions was used on Day 1 of training; another one was used on Day 2 and on retention testing. A counterbalanced paradigm was followed in which half of the subjects were trained with black cylinder as positive and the other half with gray cylinder as positive.

During the initial phase of learning this task, rats frequently put their snout close to negative cylinders and then leave them. Because olfactory cues are of no guidance in this respect, they most likely respond to the color. An approach to the positive cylinder is immediately followed by rearing and drinking from the well. As training proceeds, rats gradually cease approaching negative cylinders and head for the positive cylinder when entering the goal compartment. It is not likely that they change their learning strategy at this stage of training by addressing the positive cylinder because of its odd appearance (one positive vs. two negative cylinders), since approaching negative cylinders is seen now and then.

Statistics. Overall analyses were carried out with a one-way analysis of variance (ANOVA). Group comparisons were made with Tukey-Kramer multiple comparisons test. Computations were made with InStat statistical software program (GraphPad Software, San Diego).

\section{Results}

Histology. In the TC/LEC group, the lesions were bilateral in all animals. The fiber transections, which often affected the alveus of the hippocampus, were $0.5-1.0 \mathrm{~mm}$ long in rostrocaudal extent and 3-4 mm long in dorsoventral extent (Figures $1 \mathrm{~A}$ and $2 \mathrm{~A}$ ). In 4 rats, the ventral part of subiculum was damaged unilaterally. The mean percentage of fiber lesion was 93 (range, 82-98). Because about one-third of the fibers are not accessible for denervation, a total of about $60 \%$ of the fibers between TC and LEC were disconnected.

In the PP group, the PP was damaged bilaterally in the entire dorsoventral extent in all rats by a cut of $1.0-1.5 \mathrm{~mm}$ in length (Figures $1 \mathrm{~B}$ and $2 \mathrm{~B}$ ). The mean percentage of fiber damage was 92 (range, 88-100). Additional lesion was occasionally observed as a relatively small cut in parts of LEC and parasubiculum, and extension of the lesion site into the subiculum.

In the TC/LEC + PP group, the lesions were often seen to have fused together and were more extensive than the 
sum of separate lesions would be expected to make up (Figures 1C and 2C). The location of the separate lesions appeared very similar to those described above. The mean percentage of fiber damage was 95 for TC/LEC (range, 84-100), and 96 for PP (range, 86-100). In three rats, the ventral tip of the hippocampal region was damaged unilaterally.

In all lesioned groups, the penetrations of neocortical layers with the cannulas left hardly detectable marks. Additional damage other than that reported above could not be observed with the histological method applied.

Behavior. The groups differed both in acquisition and retention measures (Table 1). A one-way ANOVA confirmed a significant treatment effect in terms of errors during Day 1 of acquisition $[F(3,32)=9.646, p<.0001]$. Group comparisons with Tukey-Kramer multiple comparisons test showed that the PP group made reliably more errors than the control, TC/LEC, and TC/LEC + PP groups $(p<.001)$. The latter groups did not differ reliably from one another. An ANOVA revealed a significant overall effect in errors to criterion for Day $2[F(3,32)=7.410$, $p=.0007]$. The TC/LEC group made reliably more errors than both the control group, the TC/LEC + PP group ( $p<$ $.001)$, and the PP group $(p<.05)$. The latter groups were not reliably at variance. However, an ANOVA did not confirm significant differences in trials to criterion $[F(3,32)=$ $2.432, p=.0832]$.

For retention performance, an ANOVA confirmed a reliable effect in errors to criterion $[F(3,32)=10.422$, $p<.0001]$. The TC/LEC group made significantly more errors than the control, PP, and TC/LEC + PP groups $(p<.01)$. The latter groups did not differ from one another. A significant treatment effect was also seen for trials to criterion $[F(3,32)=9.840, p<.0001]$. The TC/LEC group used reliably more trials than the control, $\mathrm{PP}$, and TC/LEC + PP groups $(p<.01)$.

\section{Discussion of Experiment 1}

The results from the present experiment show that TC/ LEC lesions impaired both the acquisition (Day 2) and the retention of the discrimination task, whereas PP lesions impaired only the acquisition phase (Day 1). These results correspond with findings from previous studies employing the same lesions (see Introduction), except that PP lesions have also been seen to impair the performance on Day 2 of acquisition. However, quite unexpectedly, the combined TC/LEC + PP lesions impaired neither acquisition nor retention of the present task. This combination of lesions appeared to produce antagonistic effects rather than an accumulation of cognitive impairments.

The acquisition deficit seen in rats with TC/LEC transections seems to be of a mnemonic nature, because rats bearing such lesions did not commit more errors than control subjects on the 1st day of acquisition. This finding suggests that TC/LEC lesions interfere more with memory processing than with the ability to relearn the discrimination task. The mnemonic interpretation also receives support from the findings that TC/LEC transections impair retroactive memory more profoundly than proactive memory (Myhrer, 1992). Disruption of alreadyestablished memory engrams would be expected to have marked effects on subsequent performance, whereas subsequent performance would be expected to be less affected when memory traces are established after the lesion. The present results indicate that neither TC/LEC nor PP lesions result in a general behavioral deficit, because rats with TC/LEC lesions displayed initially normal acquisition and rats with PP lesions exhibited normal relearning.

The present finding of retention deficit following TC/ LEC lesions is reconcilable with the results of a study of mice in which damage to the distal part of the angular bundle corresponding to the level of the rhinal fissure resulted in impaired memory of a postoperatively acquired operant task (Gauthier \& Destrade, 1984). Moreover, impairment of retention, but not acquisition of the present three-choice visual discrimination test, has been seen in rats with entorhinal lesions produced by microinjections of NMDA (Levisohn \& Isacson, 1991). Also, damage to PC with mediated connections between TC and LEC results in memory deficits (see Introduction). The PP lesions caused impeded acquisition of the discrimination task, but did not affect retention of memory. Similar results have also been observed among rats with hippocampal lesions in the present discrimination task (Myhrer \& Nævdal, 1989). The hippocampal region appears to contribute more to temporary storing of information than to long-term preservation (Jarrard, 1993; Rawlins, 1985). More recently, angular bundle lesions have been reported to impair retention, but not acquisition, of three-object discrimination problems (Vnek, Gleason, Kromer, \& Rothblat, 1995). These results contrast the present ones seen after PP lesions. In the study of Vnek et al. (1995),

Table 1

\begin{tabular}{|c|c|c|c|c|c|c|}
\hline \multirow[b]{3}{*}{ Group } & \multirow[b]{3}{*}{$N$} & \multicolumn{3}{|c|}{ Acquisition } & \multirow{2}{*}{\multicolumn{2}{|c|}{ Retention }} \\
\hline & & \multirow{2}{*}{$\begin{array}{c}\text { Day } 1 \\
\text { Errors }\end{array}$} & \multicolumn{2}{|c|}{ Day 2} & & \\
\hline & & & Errors & Trials (Total) & Errors & Trials \\
\hline ontrol & 9 & $1.4 \pm 0.73$ & $0.4 \pm 0.53$ & $15.7 \pm 0.87$ & $0.4 \pm 0.53$ & $5.7 \pm 0.87$ \\
\hline $\mathrm{C} / \mathrm{LEC}$ & 9 & $1.6 \pm 0.88$ & $2.4 \pm 1.33$ & $18.7 \pm 3.67$ & $2.3 \pm 0.87$ & $11.9 \pm 3.62$ \\
\hline D & 9 & $3.3 \pm 1.00$ & $1.0 \pm 0.71$ & $17.8 \pm 1.99$ & $0.3 \pm 0.50$ & $5.6 \pm 0.88$ \\
\hline $\mathrm{C} / \mathrm{LEC}+\mathrm{PP}$ & 9 & $1.6 \pm 0.88$ & $0.4 \pm 1.33$ & $16.1 \pm 3.33$ & $0.7 \pm 1.32$ & $6.8 \pm 4.26$ \\
\hline
\end{tabular}

Note-TC, temporal cortex; LEC, lateral entorhinal cortex; PP, perforant path. 
however, the lesions encroached upon the white matter adjacent to the rhinal fissure, which may represent elements of TC/LEC transections.

The lack of effects seen to follow TC/LEC + PP lesions may be associated with alternative strategies for solving the three-choice brightness discrimination taskfor example, as a discrimination or an oddity problem. Conceivably, a partial disconnection could disrupt behavioral performance more than complete disconnection if the more lesioned animals are biased to employ an alternative strategy supported by intact brain systems. However, the present three-choice paradigm does not make rats respond to oddity (see Method), which probably would require an ability to perceive the wholeness of the situation (Myhrer, 1992). A three-choice task was used because results from previous studies have shown that hippocampal lesions affect neither acquisition nor retention of a simple two-choice brightness discrimination (Kimble, 1963; Truax \& Thompson, 1969). In agreement with such findings, hippocampal rats learn like normals when using a two-choice version of the present discrimination task (Myhrer, 1992).

The lack of effects seen after TC/LEC + PP lesions is probably not due to any combination of lesions in the temporal region. TC/LEC transections combined with hippocampal lesions result in a profound impairment in both acquiring and retaining the present discrimination task (Myhrer \& Johannesen, 1994, 1995). PP sections and hippocampal lesions produce similar acquisition deficits in the present test situation (Myhrer, 1992). The differences in behavioral effects obtained seem to be related to the combination of fiber denervations and damage to neuronal assemblies versus the combination of two types of fiber denervations.

In a previous study, we determined the pattern of terminal degeneration in the temporal region following TC/LEC transections (Myhrer et al., 1989). Degenerated terminals were predominantly seen in the superficial layers of TC, PC, and LEC, whereas the degeneration in MEC was more sparse and evenly distributed across all layers. Additional degeneration was seen in the subiculum as a result of disruption of CA1 projections in the alveus; in the parasubiculum as a result of disruption of afferents from PC; and in the superficial zone in the molecular layer of the fascia dentata as a result of disruption of lateral PP fibers at the most ventral levels. Thus, TC/ LEC lesions interrupt some extra fibers beyond those connecting TC and LEC. However, selective lesions of PC produce results similar to those seen after TC/LEC transections in the present discrimination task (Myhrer \& Wangen, 1996). Furthermore, both selective TC or LEC lesions impede visual memory (Myhrer, 1991, 1992).

Damage to LEC or MEC results in terminal degeneration in the dentate molecular layers and stratum lacunosum-moleculare of CA3 and CA1. These projections are routed in the angular bundle of the rat (HjorthSimonsen, 1972; Hjorth-Simonsen \& Jeune, 1972). Consequently, lesions of the angular bundle will effectively disrupt PP projections. However, PP lesions will also destroy entorhinal afferents originating in the hippocampal region (van Haeften, Jorritsma-Byham, \& Witter, 1995). Thus, PP transections produce not only hippocampal deafferentation, but also deefferentation. It cannot, however, be ruled out that damage to other fibers of passage may contribute to the behavioral changes seen after PP lesions.

The TC/LEC lesions and the PP lesions are localized in close vicinity. Thus, it might be argued that fibers escaped the second transection as a result of temporary deformation of tissue caused by the first transection. This was probably not the case, because the two lesions had fused together and actually made up a larger area than would be expected from each separate lesion (Figure 1).

\section{EXPERIMENT 2}

In view of the marked behavioral deficits seen to follow separate TC/LEC or PP lesions, it was rather surprising to find that the combination of these lesions did not affect learning or retention at all. The validity of this finding can be examined in several ways-for instance, histologically, neurochemically, or behaviorally. For the present experiment, it was decided to use the last alternative. Rats with combined or separate lesions were tested in a behavioral task that is supposed to activate different, and perhaps more refined, cognitive processing than that involved in the discrimination task used in Experiment 1. For this purpose, a novelty test was employed. This test has previously been shown to discriminate between effects of lateral versus medial PP lesions (Myhrer, 1988b) and medial versus lateral septal lesions (Myhrer, 1989a).

Successful performance on the simultaneous brightness discrimintaion is dependent on acquisition and retention of the principle that choice of the positive cylinder is always followed by reward. The novelty test does not involve conventional elements of reward; rather, it is based on the animals' innate tendency to explore novel objects over familiar ones. The recognition of novelty requires not only intact memory, but also preserved ability to utilize environmental cues so that changes in the environment can be detected.

Exploration of a discrete novel object is one form of inquisitive activity frequently seen among rats. This activity appears as a strong preference for novelty, the recognition of which is probably based on polymodal sensory information (Berlyne, 1960). The rats were tested in a modified version of the novelty test of Berlyne (1950), which consisted of three different sets of stimuli: visual/ tactile, olfactory, or visual only (Myhrer, 1988b).

\section{Method}

Subjects. Thirty-two male Wistar rats from the same commercial supplier (see Experiment 1), weighing 290-320 g at the time of surgery, served as subjects. They were randomly assigned to four groups: 8 rats received bilateral TC/LEC lesions, 8 received PP lesions, 8 received TC/LEC $+\mathrm{PP}$ lesions, and 8 served as controls and had only their scalp reflected. The rats were treated as described in Experiment 1. 
Surgery and histology. Surgery and histology were the same as described for Experiment 1.

Apparatus. Behavioral testing was carried out in a Plexiglas cage $(56 \times 34 \times 20 \mathrm{~cm})$ with a metal mesh roof previously described (Myhrer, 1988b). In brief, the floor was divided in 18 equal squares $(9 \times 11 \mathrm{~cm})$. Three identical metal cubes $(5 \times 5 \times 5 \mathrm{~cm})$ were evenly distributed in the cage in fixed positions (the neutral objects). Three other cubes made up the novel objects. Only one object differed from the neutral ones in that its top was uneven with tracks in it, making up a square pattern (visual/tactile stimuli). Since the rats could perceive the tracks or the squares (16 squares measuring $1 \times 1 \mathrm{~cm}$ ) by bodily contact, both tactile and visual sensory modalities might be used. One was identical with the neutral ones, and a spot of cheese (diam. $1.5 \mathrm{~cm}$ ) was smeared on the side facing the experimenter (olfactory stimulus). So-called Norwegian white cheese, which hardly smells at all to humans, was used. In the test cage, it was not possible to detect the cheese visually. One was smaller than the neutrals, and two sides were slightly uneven (visual stimulus). All objects were painted light gray. The only light was a $15-\mathrm{W}$ bulb $60 \mathrm{~cm}$ above the apparatus. The sound-attenuated testing room was provided with a fan producing white noise $(52 \mathrm{~dB})$.

Procedure. Adaption started on Postoperative Day 7. The rats were allowed to explore individually the empty apparatus for $20 \mathrm{~min}$. On the next day the rats were run in Session 1. In Phase 1, the animals were tested for $5 \mathrm{~min}$ in the box with the three neutral objects present. The following behaviors were recorded: number of seconds in contact with each object, number of squares traversed (locomotor activity), number of rearings, and duration of grooming in seconds. Then the rats spent $10 \mathrm{~min}$ in the home cage. In Phase 2, the rats were tested again for $5 \mathrm{~min}$; in this phase, one of the neutral objects had been replaced by the novel object with uneven top. During this period of time, the same measures as in Phase 1 were made. In Sessions 2 and 3 (Postoperative Days 9 and 10 ), the same procedure was followed, and the novelty was represented by a spot of cheese on one side of the cube and a smaller object, respectively. To exclude effects of positional preferences, the novel object was for each session placed in a position never used in preceding sessions. A prearranged random order for the position of novel object was made for each animal. Prior to testing, the apparatus and objects were carefully cleaned and allowed to dry.
Statistics. Statistical analyses were carried out as described for Experiment 1 .

\section{Results}

Histology. In the TC/LEC group, the lesions appeared to be very similar to those described in Experiment 1 (Figures $1 \mathrm{~A}$ and $2 \mathrm{~A}$ ). The mean percentage of fiber lesion was 91 (range, 82-100). In the PP group, the lesions were very similar to those described in Experiment 1 (Figures $1 \mathrm{~B}$ and $2 \mathrm{~B}$ ). The mean percentage of fiber lesion was 98 (range, 88-100). In the TC/LEC + PP group, the lesions also appeared like those described in Experiment 1 (Figures $1 \mathrm{C}$ and $2 \mathrm{C}$ ). The mean percentage of fiber damage was 96 (range, 92-100) for TC/LEC and for PP, it was 98 (range, 83-100). The ventral tip of the hippocampal region was damaged unilaterally in 2 rats and bilaterally in 1 rat.

Behavior. Both the TC/LEC group and the PP group displayed reduced preference for novelty compared to the control group throughout all sessions. However, the group with combined lesions (TC/LEC + PP) initially (Session 1) exhibited reduced preference for novelty, and then the preference appeared gradually more evident in Sessions 2 and 3 (Figure 3). Exploration of neutral object is based on the mean time of contact with the two neutral ones. Group comparisons were made in terms of difference between exploration of novel versus neutral objects (Table 2). For Session 1 (uneven top of novel object), a one-way ANOVA confirmed a significant treatment effect $[F(3,28)=14.702, p<.0001]$. Group comparisons with the Tukey-Kramer multiple comparisons test showed that the TC/LEC, PP, and TC/LEC + PP groups displayed reliably less preference for novelty than did the control group $(p<.001)$. The groups with lesioned animals did not differ reliably from one another. Student's $t$ test for dependent means revealed that only the control

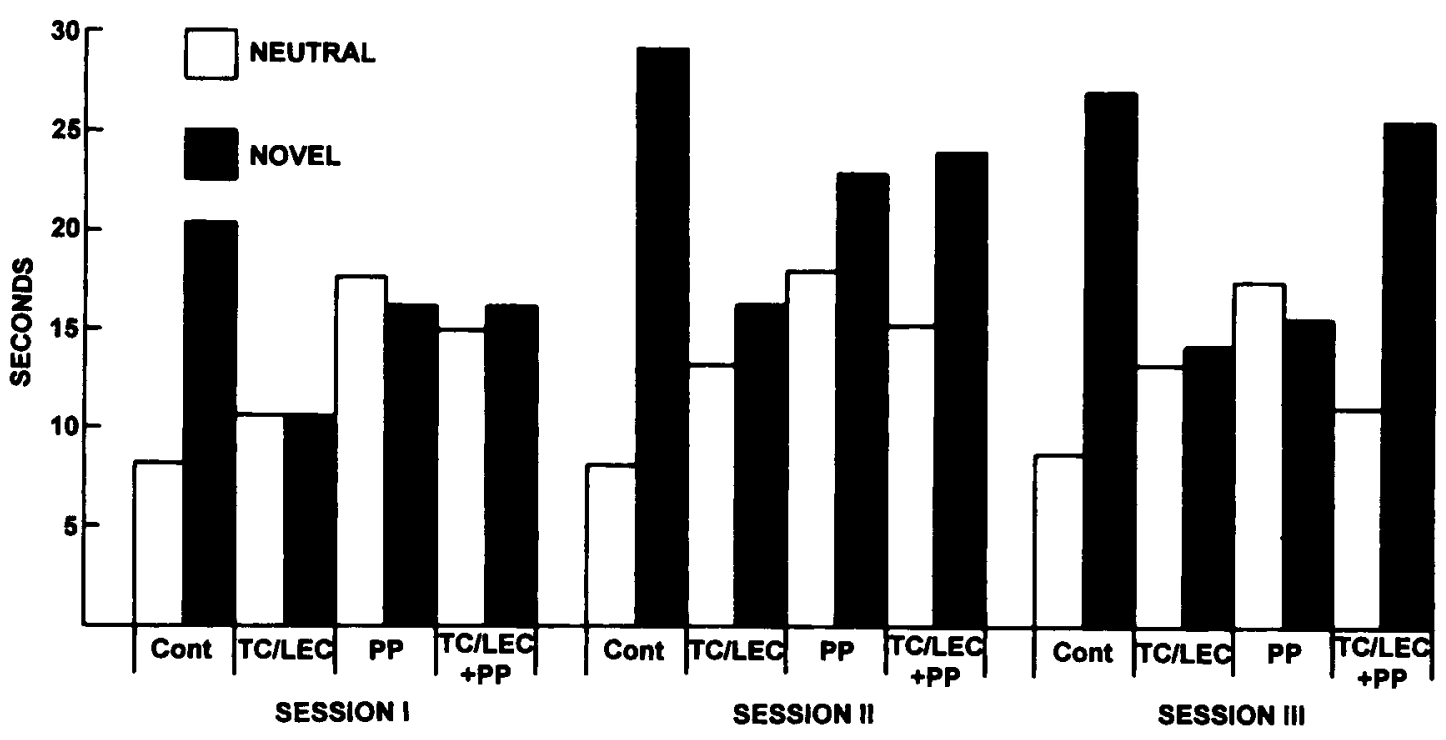

Figure 3. Mean duration of exploring objects in Experiment 2. Exploration of neutral object is based on the mean time of contact with the two neutral ones. 
Table 2

Mean Measures of Exploratory Behavior (in Seconds) in Experiment 2

\begin{tabular}{|c|c|c|c|c|c|c|c|c|c|c|c|c|c|c|c|c|}
\hline \multirow[b]{3}{*}{ Group } & \multirow[b]{3}{*}{$N$} & \multicolumn{9}{|c|}{ Differential Time Exploring } & \multicolumn{6}{|c|}{ Total Time Exploring } \\
\hline & & \multicolumn{3}{|c|}{ Session 1} & \multicolumn{3}{|c|}{ Session 2} & \multicolumn{3}{|c|}{ Session 3} & \multicolumn{2}{|c|}{ Session 1} & \multicolumn{2}{|c|}{ Session 2} & \multicolumn{2}{|c|}{ Session 3} \\
\hline & & Neut & Nov & $\overline{\text { Diff }}$ & Neut & Nov & Diff & Neut & Nov & $\overline{\text { Diff }}$ & $\mathrm{Ph} 1$ & $\mathrm{Ph2}$ & $\mathrm{Ph} 1$ & $\mathrm{Ph2}$ & Ph1 & $\mathrm{Ph} 2$ \\
\hline Control & 8 & 7.9 & 20.5 & 12.6 & 8.0 & 29.3 & 21.3 & 8.6 & 26.9 & 18.3 & 40.4 & 33.8 & 29.6 & 44.1 & 28.1 & 40.9 \\
\hline TC/LEC & 8 & 10.9 & 11.1 & 0.2 & 12.9 & 16.5 & 3.6 & 13.3 & 14.1 & 0.8 & 39.4 & 33.3 & 33.9 & 39.9 & 36.8 & 40.5 \\
\hline PP & 8 & 17.9 & 16.6 & -1.3 & 17.9 & 23.1 & 5.2 & 17.5 & 15.8 & -1.7 & 48.6 & 52.3 & 42.3 & 58.3 & 42.9 & 50.5 \\
\hline $\mathrm{TC} / \mathrm{LEC}+\mathrm{PP}$ & 8 & 15.0 & 16.6 & 1.6 & 15.4 & 24.1 & 8.7 & 11.4 & 25.6 & 14.2 & 55.6 & 46.5 & 43.9 & 54.4 & 43.5 & 47.6 \\
\hline
\end{tabular}

Note-Neut, neutral; Nov, novel; Diff, difference; Ph, phase; TC, temporal cortex; LEC, lateral entorhinal cortex; PP, perforant path.

group explored the novel object significantly more than the neutral ones $(p=.0003)$. For Session 2 (novelty smell of cheese), an ANOVA confirmed a significant overall effect in preference for novelty $[F(3,28)=10.302, p<$ $.0001]$. The TC/LEC and PP groups differed significantly from the control group $(p<.001)$. Also, the TC/LEC + PP differed from the control group $(p<.01)$. No other significant differences were seen among the groups. A $t$ test for dependent means showed that only the control group $(p<.0001)$ and the TC/LEC + PP group $(p=$ .0298 ) showed significantly more preference for the novel object than for the neutral ones. For Session 3 (smaller object), an ANOVA revealed a significant treatment effect $[F(3,28)=24.340, p<.0001]$. The TC/LEC and $\mathrm{PP}$ groups showed reliably less preference for novelty than did the control group and the TC/ LEC + PP group $(p<.001)$. No other significant group differences were observed. A $t$ test for dependent means showed that only the control group and the TC/LEC + PP group exhibited more preference for novel object versus neutral objects $(p<.0018)$.

Table 2 shows the total time spent in exploring neutral objects in Phase 1 and neutral-plus-novel objects in Phase 2. However, an ANOVA did not confirm a reliable treatment effect for any phase in any of the sessions.

The PP group tended to display more locomotor activity than did the other groups (Figure 4A). ANOVAs confirmed reliable overall effects for both phases in all three sessions $(p<.01)$. In all cases, the PP group was significantly more active than the control group and the TC/LEC group $(p<.05)$. The TC/LEC + PP group displayed locomotor activity between these extremes and was not reliably different from either the PP group or the control and TC/LEC groups.

The PP group also tended to exhibit more rearing activity than did the other groups (Figure 4B). ANOVAs confirmed significant treatment effects in all cases except Phase 1 in Session $1(p<.02)$. The PP group made reliably more rearings than did both the control and $\mathrm{TC} /$ LEC groups $(p<.05)$ except for Phase 1 in Session 2, when only the control group was significantly different from the PP group. The TC/LEC + PP group also made significantly more rearings than did the control group in Phase 2 in Sessions 2 and $3(p<.05)$. No reliable differences among the groups were seen in terms of grooming behavior.

\section{Discussion of Experiment 2}

The main results from this experiment show that all three lesion types reduced the preference for novelty. However, the effects of the TC/LEC + PP lesions became attentuated during testing. In Session 2, the preference for the novel object was partly restored, whereas it was completely reinstated in Session 3. Some remnant effects of the TC/LEC + PP lesions were seen in terms of an elevated level of rearing in Sessions 2 and 3. These results suggest that the present novelty test is a more sensitive instrument in measuring cognitive dysfunctions than the discrimination task used in Experiment 1. The suggestion is reconcilable with previous findings demonstrating differential effects of regional PP transections and regional septal lesions when using the same novelty test (Myhrer, $1988 \mathrm{~b}, 1989 \mathrm{a})$. The higher sensitivity of the novelty test may be related to the requirement of normal memorizing as well as intact ability to utilize polymodal sensory information.

Decreased preference for novelty has been observed in previous studies of rats with entorhinal or angular bundle lesions (Eriksson, Köhler, \& Sundberg, 1976; Köhler $\&$ Sundberg, 1977). In some more recent works, it has been demonstrated that perirhinal lesions reduce preference for novelty, whereas septal or fornical lesions do not (Ennaceur \& Aggleton, 1994; Ennaceur \& Meliani, 1992; Ennaceur, Neave, \& Aggleton, in press). The present results-that both TC/LEC and PP lesions interfere with the innate preference for novelty - are tenable with previous findings.

The present findings warrant the occurrence of compensatory processes in rats bearing combined lesions. The acquisition phase in Experiment 1 took place during Postoperative Days 9-10, and no cognitive deficit was recorded among the TC/LEC + PP rats. In Experiment 2, the testing took place during Postoperative Days $8-10$, and a clear deficit was seen only on Day 8 in rats with TC/LEC + PP lesions. Thus, a functional recovery appeared to start on Day 9 in the novelty test. This recovery may be associated with further experience with the test situation, although there are apparently no elements of learning involved in the novelty test. Alternatively, processes associated with neuronal plasticity reaching a peak point around Postoperative Days 9-10 may be involved in the functional recovery. A third possibility may be a combination of experiential factors and spontaneous neuronal 

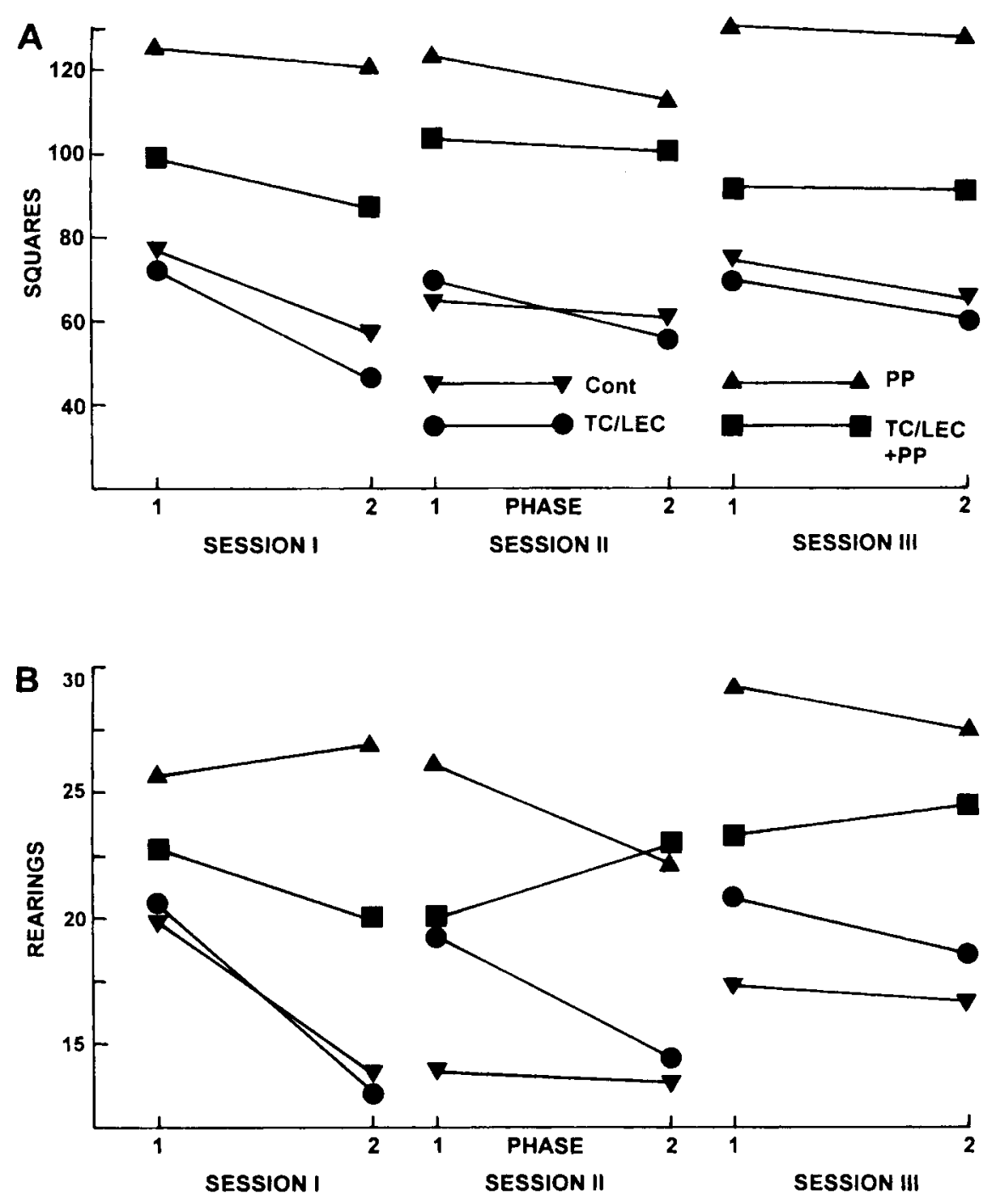

Figure 4. Mean measures of locomotor activity (A) and rearing (B) in Experiment 2.

processes supporting the gain of previously lost functions. It appears rather intriguing, however, that rats with a double lesion should profit even more effectively from environmental factors than rats bearing a single TC/LEC lesion or a single PP lesion. A fourth possibility might be that the reduced effects of TC/LEC + PP lesions are simply a result of differential sensitivity of the various test sessions. That is, the three forms of novelty are not equally salient or of similar interest to the animals. This alternative does not gain support from the present findings, because there was no preference at all for the novel object in Session 3 among rats with TC/LEC or PP lesions.

The hippocampal region is well known for compensatory responding to neural insult of areas, giving rise to hippocampal afferents. Entorhinal lesions have been reported to cause sprouting in the fascia dentata of the hippocampal region in rats within 9-12 days (Ramirez, Labbe, \& Stein, 1988). It has been proposed that this phenomenon is due to septo-dentate afferents, which can proliferate in response to entorhinal damage (Parent, Dea, Quirion, \& Poirier, 1993). The dentate sprouting has been related to amelioration of behavioral dysfunctions seen to follow unilateral entorhinal lesions. However, uncertainty exists about whether the sprouting process is entirely responsible for recovery of function when the entorhinal lesions are bilateral (Ramirez et al., 1988). If sectioning of the PP fibers causes dentate sprouting supporting the restoration of the preference for novelty, both the PP group and the TC/LEC + PP group should profit from the mitigating processes. The reasons for the differential results seen in this experiment may be elucidated by further examinations applying neurochemical analyses. The study of the strange phenomenon encountered in the present study may yield important clues in understanding processes underlying recovery of function.

Although both TC/LEC lesions and PP lesions impair recognition of novelty, different mechanisms are assumed to be involved. A deficit in memorizing may represent a 
plausible explanation of the behavior seen in TC/LEC animals. These rats may have a decreased capability to remember what objects had previously been inspected within the same phase. In this way TC/LEC animals may explore objects at random. Rats with PP lesions, on the other hand, are supposed to have reduced ability to utilize environmental cues, because the hippocampal region has strongly diminished access to sensory information of neocortical origin (Myhrer, 1988a). Elements of both deficits may be reflected in the behavior of rats with $\mathrm{TC} /$ LEC + PP lesions, since their level of locomotor activity is unchanged and their activity in rearing is increased.

\section{EXPERIMENT 3}

The unexpected recovery of function seen to occur in rats with combined TC/LEC and PP lesions may be better understood if neurochemical analyses are taken into use. Neurotransmission in TC and LEC is predominantly glutamatergic. Reduced high-affinity D-aspartate uptake in both TC and LEC is measured after TC/LEC transections (Myhrer et al., 1989), and administration of glutamatergic agonists can completely restore memory function in rats with TC/LEC lesions (Myhrer \& Paulsen, 1992). Also, the hippocampal afferents from the entorhinal cortices are believed to use glutamate as neurotransmitter (Köhler, 1986). However, the second major hippocampal input, arising in the septal area, is believed to use acetylcholine as neurotransmitter (Storm-Mathisen, 1974; Parent et al., 1993).

In response to neural injury, both glutamatergic and cholinergic systems have been shown to react in a compensatory manner. Compensatory mechanisms have been seen to normalize glutamatergic axon terminal activity in the septum following loss of innervation from the frontal cortex or the hippocampus in rats (Jaskiw, Tizabi, Lipska, Kolachana, Wyatt, \& Gilad, 1991). Entorhinal lesions have been shown to stimulate septal cholinergic afferents to proliferate into the dentate molecular layer deprived of PP input (Storm-Mathisen, 1974). Thus, glutamatergic and cholinergic systems in the temporalhippocampal region seem to be the most relevant transmitter systems to be examined in this experiment.

\section{Method}

Subjects. Thirty male Wistar rats from the same commercial supplier (see Experiment 1), weighing 290-320 g at the time of surgery, served as subjects. They were randomly assigned to four groups: 8 rats received bilateral TC/LEC lesions, 7 received PP lesions, 8 received TC/LEC + PP lesions, and 7 served as controls and had only their scalp reflected. The rats were treated as described in Experiment 1.

Surgical procedures. Surgical procedures were the same as those described for Experiment 1.

Neurochemistry. For uptake and enzyme activity studies, the rats were decapitated on Postoperative Day 14, and the brains were removed. Samples from LEC (Broadmann's area 28b), TC (Broadmann's areas 20,36, 41), and the hippocampal region (including hippocampus proper, fascia dentata, and subiculum) were dissected out from each hemisphere. The tissue samples were homogenized in cold $0.32-\mathrm{M}$ sucrose with a glass-Teflon homog- enizer. The homogenates were kept cold until measures of highaffinity uptake and enzyme assays were conducted. All determinations were performed in triplicate.

High-affinity D-aspartate uptake was chosen as the marker for glutamatergic nerve terminals (Fonnum, 1984). The determination of high-affinity D-aspartate uptake has previously been described (Lund Karlsen \& Fonnum, 1978). Choline acetyltransferase (ChAT) was chosen as the marker for cholinergic nerve terminals. Previously described methods were used for determination of ChAT (Fonnum, 1975). Protein content was measured with the method of Lowry, Rosebrough, Farr, and Randell (1951).

\section{Results}

Neurochemistry. Several effects of the lesions were seen in the neurochemical measures (Tables 3 and 4). An ANOVA confirmed a significant overall effect in Daspartate uptake in the TC $[F(3,26)=17.845, p<.0001]$. Tukey-Kramer post hoc comparisons showed that both the TC/LEC and TC/LEC + PP groups had significantly lower uptake of D-aspartate than did the control group $(p<.001)$ and the PP group $(p<.001)$. An ANOVA did not reveal reliable differences in uptake of $D$-aspartate in the lateral entorhinal cortex or the hippocampal region. However, a marked asymmetrical distribution of Daspartate uptake was seen in both the TCs and LECs of the control animals. A $t$ test for dependent means showed that the left TC contained higher levels of D-aspartate than the right TC $(p=.0067)$. A similar asymmetry was also seen between left and right LECs $(p=.0005)$.

Table 4 shows that responses to the lesions were seen only in terms of ChAT levels in the hippocampal region. An ANOVA confirmed a reliable treatment effect $[F(3,26)$ $=3.713, p=.0240]$. Group comparisons showed that the PP group had significantly lower levels of ChAT than the TC/LEC + PP group only $(p<.05)$. Because the distribution of ChAT across the left and right hippocampi was even, it appeared justified to analyze all single observations together. An ANOVA showed a significant overall effect in the hippocampal region $[F(3,56)=5.635$, $p=.0019]$. The levels of ChAT were reliably lower in the PP group than in the control group $(p<.01)$, the TC/ LEC group $(p<.05)$, and the TC/LEC + PP group ( $p<$ $.01)$.

\section{Discussion of Experiment 3}

The main results from the present experiment show that the levels of ChAT in the hippocampal region differed among the lesioned groups. PP lesions resulted in a decrease of ChAT in the hippocampal region, whereas TC/LEC and TC/LEC + PP lesions did not lower hippocampal ChAT concentrations. The levels of high-affinity D-aspartate uptake also differed among the groups. Both TC/LEC and TC/LEC + PP lesions reduced D-aspartate uptake in TC. Among the control animals, a clear asymmetrical distribution of D-aspartate uptake (higher in the left hemisphere) was seen in both TC and LEC, but was not evident in the hippocampal region.

The levels of ChAT can be interpreted as reflecting assemblies of nerve terminals containing acetylcholine, and the levels of D-aspartate uptake may reflect assem- 
Table 3

Mean Levels of High-Affinity D-Aspartate Uptake (pmol/min/ $/ \mathrm{g}$ protein) in Experiment 3

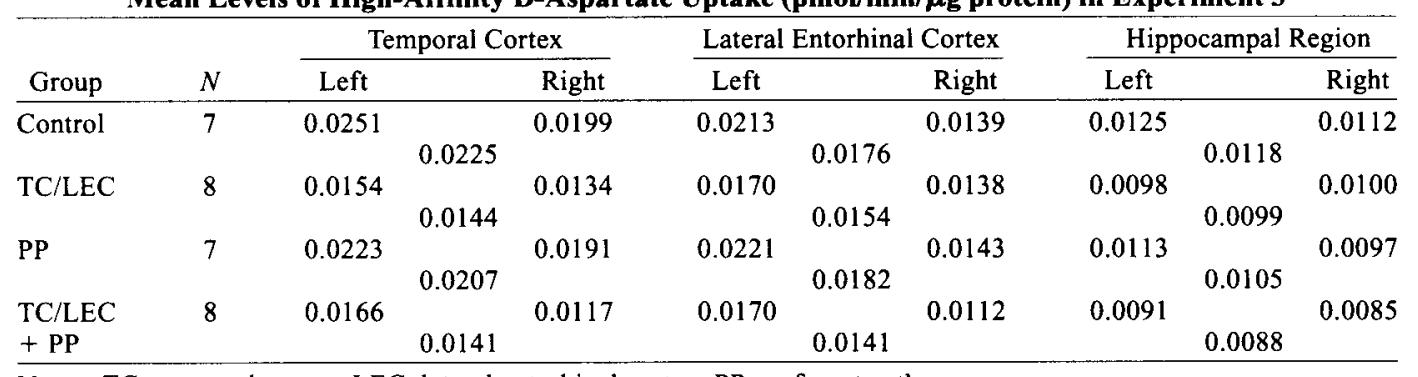

Note-TC, temporal cortex; LEC, lateral entorhinal cortex; PP, perforant path.

Table 4

Mean Levels of Choline Acetyltransferase (nmol/min/mg protein) in Experiment 3

\begin{tabular}{|c|c|c|c|c|c|c|c|c|c|c|}
\hline \multirow[b]{2}{*}{ Group } & \multirow[b]{2}{*}{$N$} & \multicolumn{3}{|c|}{ Temporal Cortex } & \multicolumn{3}{|c|}{ Lateral Entorhinal Cortex } & \multicolumn{3}{|c|}{ Hippocampal Region } \\
\hline & & Left & & Right & Left & & Right & Left & & Right \\
\hline Control & 7 & 0.7176 & 0.6764 & 0.6352 & 0.7352 & 0.6911 & 0.6470 & 0.7294 & 0.7617 & 0.7941 \\
\hline TC/LEC & 8 & 0.5058 & 0.5440 & 0.5823 & 0.7058 & 0.7117 & 0.7176 & 0.6705 & 0.7381 & 0.8058 \\
\hline PP & 7 & 0.6647 & 0.6441 & 0.6235 & 0.5588 & 0.6000 & 0.6411 & 0.5294 & 0.5441 & 0.5588 \\
\hline $\begin{array}{l}\text { TC/LEC } \\
+\mathrm{PP}\end{array}$ & 8 & 0.6117 & 0.5852 & 0.5588 & 0.6235 & 0.6441 & 0.6647 & 0.7588 & 0.7852 & 0.8117 \\
\hline
\end{tabular}

Note-TC, temporal cortex; LEC, lateral entorhinal cortex; PP, perforant path.

blies of nerve terminals containing glutamate. In view of the neurochemical results, the paradoxical lack of behavioral effects seen in rats with TC/LEC + PP transections may be related to the maintained levels of cholinergic nerve terminals in the hippocampal region, because reduced cholinergic activity in the hippocampal region caused by PP lesions alone would also be expected to occur in combination with TC/LEC lesions. Glutamatergic systems did not seem to contribute to the behavioral recovery observed in TC/LEC + PP animals. Actually, a tendency to decreased levels of glutamate was seen in all areas examined in the latter rats (Table 3 ).

Marked increases in acetylcholinesterase (AChE) staining or activity are seen in the molecular layer of fascia dentata following disruption of the PP input (Cotman, Matthews, Taylor, \& Lynch, 1973; Lynch, Matthews, Mosko, Parks, \& Cotman, 1972; Scheff, Benardo, \& Cotman, 1980; Stanfield \& Cowan, 1982; Storm-Mathisen, 1974). This finding contrasts the present result-that $P P$ lesions caused decreased levels of ChAT in the hippocampal region. However, increased AChE staining can occur without a corresponding change in ChAT levels in the hippocampus (Aubert, Poirier, Gauthier, \& Quirion, 1994; McKeon, Vietje, \& Wells, 1989). An increase of AChE staining in the hippocampal molecular layer is still observed in animals with combined lesions of the septum and entorhinal cortex (Chen, van Hoesen, Barnes, \& West, 1983). Furthermore, multiple cholinergic markers are not altered in response to unilateral entorhinal lesions, and it has been suggested that the increase in $\mathrm{AChE}$ staining in the molecular layer of fascia dentata after entorhinal lesions may be due to changes in noncholinergic neurons (Aubert et al., 1994). Following unilateral entorhinal lesions, unchanged values of ChAT have been measured in the entire ipsilateral hippocampus (Aubert et al., 1994). In view of the latter finding, the present decreased ChAT activity seen in assays made from the entire hippocampal region following bilateral PP transections may not appear that unexpected.

Following transection of the fimbria, a decrease in ChAT of about $90 \%$ has been seen in the hippocampal region, and in the subfield CA1 the decrease is only $70 \%$ to $80 \%$ (Storm-Mathisen, 1972). These findings suggest that cholinergic fibers also innervate the hippocampal region by way of another route than the fimbria. The angular bundle may represent an alternative route, since PP lesions caused reduced levels of ChAT in the hippocampal region. Why an additional lesion should result in unchanged levels of cholinergic activity in TC/LEC + PP animals relative to control rats is not obvious. It may be that the TC/LEC lesion and the PP lesion together transected a sufficient number of cholinergic fibers (or other fibers) in the white matter to trigger a compensatory hippocampal reinnervation. Such a reinnervation may occur by way of the cholinergic projection of the medial septal nuclei (Wenk, Bigl, \& Meyer, 1980).

The growth impact of cholinergic fibers has further been demonstrated in rats with embryonic septal transplants implanted into the fimbria. Additional lesion of the PP stimulates the ingrowth of cholinergic axons into the cholinergically denervated hippocampus. The axons of the cholinergic neurons in the implant grow both at a 
faster rate and for a longer period of time (Björklund, Kromer, \& Stenevi, 1979). The integrity of cholinergic afferents to the hippocampal region has proved important for learning and memory. Damage to the medial septal area results in decreased levels of ChAT in the hippocampus and impaired working memory in rats (Hepler, Olton, Wenk, \& Coyle, 1985). However, cholinergic systems may be more related to attentional processes than to learning and memory per se (Robbins et al., 1989). The recovery of function seen in rats with TC/LEC + PP lesions may be associated with the finding of normal level of hippocampal cholinergic activity in these animals, since PP lesions alone result in decreased cholinergic activity in the hippocampal region. This finding, however, does not preclude compensatory changes in other transmitter systems or an upregulation of peptidergic pathways playing in concert with cholinergic compensatory activity.

Histological verification of the lesions was not reconcilable with the need to dissect out relevant areas of tissue in the present experiment. However, the types of surgery used in this study produce very consistent lesions. No animals in Experiments 1 or 2 were discarded because of unsatisfactory lesions.

\section{GENERAL DISCUSSION}

The present set of experiments has shown that separate TC/LEC transections or PP transections both interfere with visual discrimination, whereas a combination of these lesions does not affect behavior in the same task. Further examination with a novelty test showed that an initial effect of TC/LEC + PP lesions disappeared during testing, whereas separate lesions yielded effects throughout the entire testing period. Neurochemical changes seen to follow the various types of lesions suggest that a compensatory optimal level of cholinergic activity in the hippocampal region may be associated with the recovery of function seen in animals bearing the double lesion.

Sprouting of cholinergic nerve terminals in the hippocampal region has been associated with recovery of function in rats with entorhinal lesions (Ramirez et al., 1988). Parallel features are seen in the time course for behavioral recovery among TC/LEC + PP animals in the novelty test and previously reported occurrence of cholinergic response in the fascia dentata following entorhinal lesion. Recovery of the preference for novelty seemed to start on Postoperative Day 9 and was complete on Day 10 in rats with TC/LEC + PP lesions. Entorhinal lesions functionally similar to TC/LEC + PP lesions have been reported to increase ChAT activity in the fascia dentata, reaching a peak point at 8 days of survival (Storm-Mathisen, 1974). The combination of TC/LEC and PP lesions appears to counteract the loss of cholinergic terminals seen to follow separate PP transections. The functional recovery in TC/LEC + PP rats is probably related to an atropinesensitive mechanism, because administration of atropine to rats that have fully recovered the preference for nov- elty causes reappearance of the lesion effect (Myhrer, unpublished data). This finding, however, cannot be causally related to the hippocampal region. Furthermore, the maintained level of hippocampal ChAT activity in rats with combined lesions does not indicate that the activity of cholinergic neurons is normal. ChAT activity reflects neuronal integrity and not manufacturing or release of acetylcholine.

The decreased level of glutamate in TC seen in rats with TC/LEC transections appears to be related to behavior in a meaningful way, because TC/LEC animals displayed both impaired acquisition and retention of the discrimination task and reduced ability to recognize environmental changes. A corresponding decrease of glutamate in TC of rats with the double lesion may be related to the initial deficit on the novelty task. No compensatory optimal level of glutamate in TC was seen in response to the combined TC/LEC and PP lesions.

\section{REFERENCES}

Aubert, I., Poirier, J., Gauthier, S., \& Quirion, R. (1994). Multiple cholinergic markers are unexpectedly not altered in the rat dentate gyrus following entorhinal cortex lesions. Journal of Neuroscience, 14, 2476-2484.

BERLYNE, D. E. (1950). Novelty and curiosity as determinants of exploratory behaviour. British Journal of Psychology, 41, 68-80.

BerLYNE, D. E. (1960). Conflict, arousal, and curiosity. New York: McGraw-Hill.

Björklund, A., Kromer, L. F., \& Stenevi, U. (1979). Cholinergic reinnervation of the rat hippocampus by septal implants is stimulated by perforant path lesion. Brain Research, 173, 57-64.

BRAAK, H., \& BRAAK, E. (1991). Neuropathological staging of Alzheimer-related changes. Acta Neuropathologica, 82, 239-259.

Chen, L.-L., van Hoesen, G. W., Barnes, C. L., \& West, J. R. (1983). Enhanced acetylcholinesterase staining in the hippocampal perforant pathway zone after combined lesions of the septum and entorhinal cortex. Brain Research, 272, 354-359.

Cotman, C. W., Matrhews, D. A., Taylor, D., \& Lynch, G. (1973). Synaptic rearrangement in the dentate gyrus: Histochemical evidence of adjustments after lesions in immature and adult rats. Proceedings of the National Academy of Sciences, 70, 3473-3477.

Deacon, T. W., Eichenbaum, H., Rosenberg, P., \& Eckman, K. W. (1983). Afferent connections of the perirhinal cortex in the rat. Journal of Comparative Neurology, 220, 168-190.

EnNaCEur, A., \& Aggleton, J. P. (1994). Spontaneous recognition of object configurations in rats: Effects of fornix lesions. Experimental Brain Research, 100, 85-92.

ENNACEUR, A., \& Meliani, K. (1992). A new one-trial test for neurobiological studies of memory in rats. III. Spatial vs. non-spatial working memory. Behavioural Brain Research, 51, 83-92.

Ennaceur, A., Neave, N., \& Aggleton, J. P. (in press). Neurotoxic lesions of the perirhinal cortex do not mimic the behavioural effects of fornix transections in the rat. Behavioural Brain Research.

ERIKSSON, H. E., KöHLER, C., \& SUNDbERG, H. (1976). Exploratory behavior after angular bundle lesions in the albino rat. Behavioral $B i-$ ology, 17, 123-130.

FonNUM, F. (1975). A rapid radiochemical method for the determination of choline acetyltransferase. Journal of Neurochemistry, 24, 407-409.

FonNum, F. (1984). Glutamate: A neurotransmitter in mammalian brain. Journal of Neurochemistry, 42, 1-11.

Gauthier, M., \& Destrade, C. (1984). Late post-learning effect of entorhinal cortex electrical stimulation persists despite destruction of the perforant path. Brain Research, 310, 174-179. 
Hepler, D. J., Olton, D. S., Wenk, G. L., \& Coyle, J. T. (1985). Lesions in nucleus basalis magnocellularis and medial septal area of rats produce qualitatively similar memory impairments. Journal of Neuroscience, 5, 866-873.

Huorth-Simonsen, A. (1972). Projection of the lateral part of the entorhinal area to the hippocampus and fascia dentata. Journal of Comparative Neurology, 146, 219-231.

HJoRTh-Simonsen, A., \& JeUne, B. (1972). Origin and termination of the hippocampal perforant path in the rat studied by silver impregnation. Journal of Comparative Neurology, 144, 215-231.

JARRARD, L. E. (1993). On the role of the hippocampus in learning and memory in the rat. Behavioral \& Neural Biology, 60, 9-26.

JASKiW, G. E., Tizabi, Y., LiPSKa, B. K., Kolachana, B. S., Wyatt, R. J., \& GilAD, G. M. (1991). Evidence for a frontocortical-septal glutamatergic pathway and compensatory changes in septal glutamate uptake after cortical and fornix lesions in the rat. Brain Research, 550, 7-10.

KimbLe, D. P. (1963). The effects of bilateral hippocampal lesions in rats. Journal of Comparative \& Physiological Psychology, 56, 273283.

KöHLER, C. (1986). Cytochemical architecture of the entorhinal area. In R. Schwarcz \& Y. Ben-Ari (Eds.), Excitatory amino acids and epilepsy (pp. 83-98). New York: Plenum.

KöHLER, C., \& SUNDBERG, H. (1977). Locomotor activity and exploratory behavior after medial entorhinal cortex lesions in the albino rat. Behavioral Biology, 20, 419-432.

Kosel, K. C., Van Hoesen, G. W., \& Rosene, D. L. (1982). Nonhippocampal cortical projections from the entorhinal cortex in the rat and the rhesus monkey. Brain Research, 244, 201-213.

LevisoHn, L. F., \& Isacson, O. (1991). Excitotoxic lesions of the rat entorhinal cortex. Effects of selective neuronal damage on acquisition and retention of a non-spatial reference memory task. Brain Research, 564, 230-244.

Lowry, O. H., Rosebrough, N. J., Farr, A. L., \& Randell, R. J. (1951). Protein measurement with the folin phenol reagent. Journal of Biological Chemistry, 193, 265-275.

LUnd Karlsen, R., \& Fonnum, F. (1978). Evidence for glutamate as neurotransmitter in the corticofugal fibres to the dorsal lateral geniculate body and the superior colliculus in rats. Brain Research, 151, 457-467.

Lynch, G., Matthews, D. A., Mosko, S., Parks, T., \& Cotman, C. (1972). Induced acetylcholinesterase-rich layer in rat dentate gyrus following entorhinal lesions. Brain Research, 42, 311-318.

McKeon, R. J., Vietje, B. P., \& Wells, J. (1989). Increase in acetylcholinesterase in the molecular layer of the dentate gyrus in the absence of septal inputs following selective granule cell lesions. Brain Research, 503, 317-321.

Mumby, D. G., \& Pinel, P. J. (1994). Rhinal cortex lesions and object recognition in rats. Behavioral Neuroscience, 108, 11-18.

MYHRER, T. (1988a). Exploratory behavior and reaction to novelty in rats with hippocampal perforant path systems disrupted. Behavioral Neuroscience, 102, 356-362.

MyhreR, T. (1988b). The role of medial and lateral hippocampal perforant path lesions and object distinctiveness in rats' reaction to novelty. Physiology \& Behavior, 42, 371-377.

MyHRER, T. (1989a). Exploratory behavior and reaction to novelty: Effects of medial and lateral septal lesions. Behavioral Neuroscience, 103, 1226-1233.

MYHRER, T. (1989b). Exploratory behavior, reaction to novelty, and proactive memory in rats with temporo-entorhinal connections disrupted. Physiology \& Behavior, 45, 431-436.

MYHRER, T. (1991). Retroactive memory of a visual discrimination task in the rat: Role of temporal-entorhinal cortices and their connections. Experimental Brain Research, 84, 517-524.

MYHRER, T. (1992). Selective lesions in the temporal-hippocampal region of the rat: Effects on acquisition and retention of a visual discrimination task. Behavioral \& Neural Biology, 58, 8-15.

Myhrer, T., IVersen, E. G., \& Fonnum, F. (1989). Impaired reference memory and reduced glutamergic activity in rats with temproentorhinal connections disrupted. Experimental Brain Research, $77,499-506$.
Myhrer, T., \& Johannesen, T. S. (1994). Cognitive and mnemonic dysfunctions in rats with hippocampal-entorhinal lesions: Attenuating effects of glycine injections. Psychobiology, 22, 61-67.

MYHRER, T., \& JoHANNESEN, T. S. (1995). Learning and retention of a visual discrimination task in rats with various combinations of lesions in the temporal-hippocampal region. Brain Research Bulletin, 36, 499-503.

Myhrer, T., \& NÆvdal, G. A. (1989). The temporal-hippocampal region and retention. The role of temporo-entorhinal connections in rats. Scandinavian Journal of Psychology, 30, 72-80.

Myhrer, T., \& Paulsen, R. E. (1992). Memory dysfunction following disruption of glutamergic systems in the temporal region of the rat: Effects of agonistic amino acids. Brain Research, 599, 345-352.

MYhrer, T., \& WANGEN, K. (1996). Marked retrograde and anterograde amnesia of a visual discrimination task in rats with selective lesions of the perithinal cortex. Neurobiology of Learning \& Memory, 65, 244-252.

Nagahara, A. H., Otto, T., \& Gallagher, M. (1995). Entorhinalperirhinal lesions impair performance of rats on two versions of place learning in the Morris water maze. Behavioral Neuroscience, 109, 3-9.

Otтo, T., \& Eichenbaum, H. (1992). Complementary roles of the orbital prefrontal cortex and the perirhinal-entorhinal cortices in an odor-guided delayed-nonmatching-to-sample task. Behavioral Neuroscience, 106, 762-775.

Parent, A., Dea, D., Quirion, R., \& Polrier, J. (1993). [ ${ }^{3}$ H] Phorbol ester binding sites and neuronal plasticity in the hippocampus following entorhinal cortex lesions. Brain Research, 607, 23-32.

RamireZ, J. J., LABBE, R., \& Stein, D. G. (1988). Recovery from perseverative behavior after entorhinal cortex lesions in rats. Brain Research, 459, 153-156.

RaWLinS, J. N. P. (1985). Associations across time: The hippocampus as a temporary memory store. Behavioral \& Brain Sciences, 8,479 497.

Robbins, T. W., Everitt, B. J., Marston, H. M., Wilkinson, J., Jones, G. H., \& PAGE, K. J. (1989). Comparative effects of ibotenic acidand quisqualic acid-induced lesions of the substantia innominata on attentional function in the rat: Further implications for the role of cholinergic neurons of the nucleus basalis in cognitive processes. Behavioural Brain Research, 35, 22 1-240.

SchefF, S. W., Benardo, L. S., \& Cotman, C. W. (1980). Decline in reactive fiber growth in the dentate gyrus of aged rats compared to young adult rats following entorhinal cortex removal. Brain Research, 199, 21-38.

STANFIELD, B. B., \& CowAN, W. M. (1982). The sprouting of septal afferents to the dentate gyrus after lesions of the entorhinal cortex in adult rats. Brain Research, 232, 162-170.

STORM-MATHISEN, J. (1972). Glutamate decarboxylase in the rat hippocampal region after lesions of the afferent fibre systems. Evidence that the enzyme is localized in intrinsic neurons. Brain Research, 40, 215-235.

StORM-MATHISEN, J. (1974). Choline acetyltransferase and acetylcholinesterase in fascia dentata following lesion of the entorhinal afferents. Brain Research, 80, 181-197.

Swanson, L. W., \& Köhler, C. (1986). Anatomical evidence for direct projections from the entorhinal area to the entire cortical manthe in the rat. Journal of Neuroscience, 6, 3010-3023.

Truax, T., \& Thompson, R. (1969). Role of the hippocampus in performance of easy and difficult visual discrimination tasks. Journal of Comparative \& Physiological Psychology, 67, 228-234.

van Haeften, T., Jorritsma-Byham, B., \& Witter, M. P. (1995). Quantitative morphological analysis of subicular terminals in the rat entorhinal cortex. Hippocampus, 5, 452-459.

VAyssettes-Courchay, C., \& Sessler, F. M. (1983). Neurophysiologie: Mise en évidence de convergences sensorielles dans le cortex entorhinal du Rat. Comptes Rendues de L'Académie des Sciences de Paris, 296, 877-879.

Vnek, N., Gleason, T. C., Kromer, L. F., \& Rothblat, L. A. (1995). Entorhinal-hippocampal connections and object memory in the rat: Acquisition versus retention. Journal of Neuroscience, 15, 3193. 3199. 
VoGT, B. A. (1974). A reduced silver stain for normal axons in the central nervous system. Physiology \& Behavior, 13, 837-840.

WENK, H., BigL, V., \& MEYER, U. (1980). Cholinergic projections from magnocellular nuclei of the basal forebrain to cortical areas in the rat. Brain Research Reviews, 2, 295-316.

WIIG, K. A., \& BILKEY, D. K. (1994a). The effects of perirhinal cortical lesions on spatial reference memory in the rat. Behavioural Brain Research, 63, 101-109.
Wirg, K. A., \& Bilkey, D. K. (1994b). Perirhinal cortex lesions in rats disrupt performance in a spatial DNMS task. Neuroreport, $5,1405-$ 1408.

(Manuscript received March 26, 1996; revision accepted for publication May 9, 1996.) 